\section{REFERENCES}

1 Blackmon SH, Rice DC, Correa AM, et al. Management of primary pulmonary artery sarcomas. Ann Thorac Surg 2009; 87: 977-984.

2 Cox JE, Chiles C, Aquino SL, et al. Pulmonary artery sarcomas: a review of clinical and radiologic features. J Comput Assist Tomogr 1997; 21: 750-755.

3 Kauczor HU, Schwickert HC, Mayer E, et al. Pulmonary artery sarcoma mimicking chronic thromboembolic disease: computed tomography and magnetic resonance imaging findings. Cardiovasc Intervent Radiol 1994; 17: 185-189.

4 Rafal RB, Nichols JN, Markisz JA. Pulmonary artery sarcoma: diagnosis and postoperative follow-up with gadolinium-diethylenetriamine pentaacetic acid-enhanced magnetic resonance imaging. Mayo Clin Proc 1995; 70: 173-176.

5 Kacl GM, Bruder E, Pfammatter T, et al. Primary angiosarcoma of the pulmonary arteries: dynamic contrast-enhanced MRI. J Comput Assist Tomogr 1998; 22: 687-691.
6 Thurer RL, Thorsen A, Parker JA, et al. FDG imaging of a pulmonary artery sarcoma. Ann Thorac Surg 2000; 70: 1414-1415.

7 Chong S, Kim TS, Kim BT, et al. Pulmonary artery sarcoma mimicking pulmonary thromboembolism: integrated FDG PET/ CT. AJR Am J Roentgenol 2007; 188: 1691-1693.

8 Wittram C, Scott JA. ${ }^{18}$ F-FDG PET of pulmonary embolism. AJR Am J Roentgenol 2007; 189: 171-176.

9 Bastiaannet E, Groen H, Jager PL, et al. The value of FDG-PET in the detection, grading and response to therapy of soft tissue and bone sarcomas; a systematic review and meta-analysis. Cancer Treat Rev 2004; 30: 83-101.

10 Farsad M, Pernter P, Triani A, et al. Thromboembolism in pulmonary artery sarcoma. Clin Nucl Med 2009; 34: 239-240.

DOI: 10.1183/09031936.00114708

\title{
Glomus vagale presenting as chronic cough
}

\section{To the Editors:}

Chronic cough is one of the most common symptoms of medical importance with $12 \%$ of the population reporting significant symptoms on a daily or weekly basis. The differential diagnosis of chronic cough is extensive. Uncommon causes of chronic cough may be missed unless an adequate history is obtained, a detailed examination performed and appropriate investigations arranged. Herein, we present a case of chronic cough, the aetiology of which was previously unreported.

A 57-yr-old female presented with a history of chronic cough for 12 months. The cough had features of reflux [1] as it was precipitated by food and phonation, and associated with frequent clearing of the throat. Moreover, the cough was worse when she was lying flat and started upon rising in the morning. In addition, she described her cough to be precipitated by upward movement of her neck, for example looking at the top shelf in a supermarket. There was no history of decreased appetite or weight loss. She was known to have a hiatus hernia. Her past medical history was otherwise unremarkable. She was a life long nonsmoker and was not on any regular medications. In view of clinical history being highly suggestive of reflux cough, she was started on treatment with lansoprazole in combination with ranitidine; however, she did not respond.

On the second consultation, fullness on the right side of her neck was observed. On examination, a prominent, distended right jugular vein was observed, suggesting the possibility of vascular lesion in the neck. A computed tomography (CT) scan of the neck revealed a brightly enhancing mass of the carotid sheath splaying the internal carotid artery and the internal jugular vein (fig. 1a). Magnetic resonance imaging (MRI) confirmed the CT scan findings of a lobulated and well circumscribed mass measuring $2 \times 2 \times 3.5 \mathrm{~cm}$ in diameter extending from just above the carotid bifurcation to just below the skull base. The lesion enhanced significantly following gadolinium injection. Magnetic resonance angiography (MRA) demonstrated multiple vessels within the lesion (fig. 1b). In addition, there appeared to be redundancy of the right aryepiglottic fold with slight medial displacement of the right vocal cord suggestive of recurrent laryngeal nerve palsy. These radiographic features suggested the mass was a "glomus vagale". Surgical resection of the tumour was successfully undertaken following pre-operative embolisation. The histology from the tumour showed a mixture of trabecular and nested patterns of cells with strongly positive staining to S-100, synaptophysin and chromogranin, consistent with a diagnosis of vagal paraganglioma. Investigations for systemic associations revealed normal urinary catecholamine levels. Postoperatively, she developed a hoarse voice and worsening regurgitation demonstrating expected vagal damage during surgical resection. However, coughing secondary to neck movement completely ceased following the removal of the tumour.
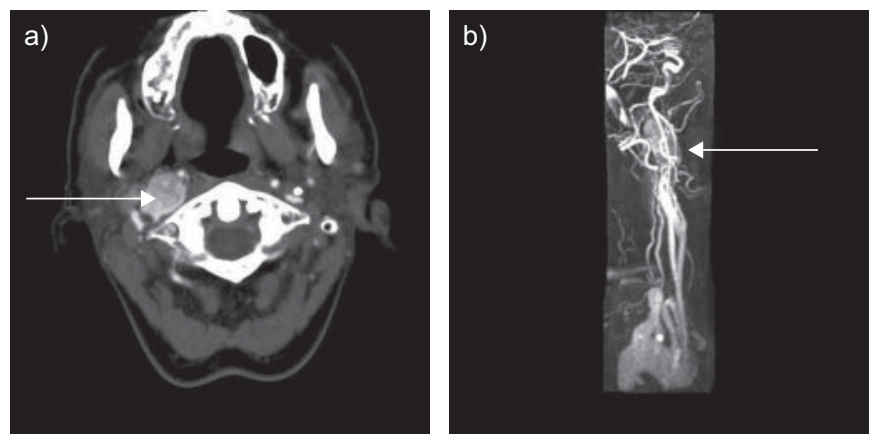

FIGURE 1. a) Contrast-enhanced computed tomography image of the neck showing a brightly enhancing mass (arrow) separating the internal carotid and internal jugular vein on the right side. b) Contrast-enhanced magnetic resonance angiography demonstrating multiple vessels within the mass (arrow) along the right carotid sheath extending from just above the carotid bifurcation to just below the skull base. 
Herein, we report a very rare case of glomus vagale associated cough. This patient had two aspects to her cough. First, a chronic cough probably associated with gastro-oesophageal reflux and secondly, a component due to the glomus tumour exemplified by precipitation of cough by upward neck movements suggesting local mechanical effect of the tumour. In addition, it is possible that there was hypersensitivity of the vagus nerve secondary to the tumour.

Vagal paragangliomas are the tumours arising from paraganglionic tissue along the vagus nerve. The paragangliomas in the head and neck can be found in four primary locations which include the jugular bulb, the middle ear cavity, the vagus nerve and the carotid body. The majority of paragangliomas are benign and malignant behaviour is seen in $<10 \%$ of cases [2, 3]. Vagal paragangliomas generally present as a painless mass in the upper neck. There may be associated hoarseness of voice due to compression of vagus nerve. Horner's syndrome may also be seen secondary to involvement of cervical sympathetic nerves. Depending on the location of the paraganglioma, other symptoms may include pulsatile tinnitus, vertigo, hearing loss and dysphagia. In our case the presentation was consistent with that of a highly vascular paraganglioma leading to venous engorgement.

A CT scan may be limited in providing soft tissue details at the skull base. MRI with gadolinium enhancement provides enhanced imaging, delineating the relationship of glomus tumours with the skull base and adjacent vascular structures. MRA may be helpful in patients undergoing pre-operative evaluation for surgical resection and help plan intravascular embolisation prior to resection. However, digital subtraction angiography may be superior to MRA for vascular assessment of these tumours [4]. Immunohistochemistry can be a useful means to confirm the diagnosis of paragangliomas in addition to routine histology and may provide information regarding the probable prognosis [5].

The preferred treatment modality in malignant paragangliomas is surgical resection [6]. It requires a multidisciplinary approach in carefully selected cases to achieve complete surgical excision [7]. The factors that should be taken into account prior to surgical resection are the patient's age, tumour size and site, pre-existing cranial nerve deficit and patient preference. In a review of 46 patients with vagal paragangliomas, NeTTERVILLE et al. [7] reported complications associated with damage to cervical sympathetics or cranial nerves in more than half of the cases. Gastrointestinal dysfunction, manifesting as persistent nausea, vomiting and regurgitation was seen due to vagal damage, while facial pain in the parotid region with the first bite of each meal (called first bite syndrome) was attributed to loss or damage of cervical sympathetics. The alternative to surgical resection is radiation therapy. It should be reserved for elderly patients and the patients who are at particular risk of bilateral cranial nerve deficits. Moreover, radiotherapy can be used as an adjunct following surgical removal of malignant paraganglioma to improve local and distant spread via lymphatics [6].

To our knowledge, this is the first case of chronic cough associated with glomus vagale tumour. This case illustrates the importance of careful clinical history and examination in the evaluation of cough. Furthermore, it gives us an insight into understanding unusual cause of cough secondary to local mechanical pressure on the vagus nerve. Although chronic cough itself is very common, this case illustrates that there can be a very rare associated cause as well.

\section{A. Fahim*, S. Faruqi* , N.D. Stafford ${ }^{\#}$ and A.H. Morice*}

*Dept of Cardiovascular and Respiratory Studies, Castle Hill Hospital, Cottingham, and "Dept of Otorhinolaryngology and Head and Neck Surgery, Hull Royal Infirmary, Hull, UK.

Correspondence: A. Fahim, Dept of Cardiovascular and Respiratory Studies, Castle Hill Hospital, Castle Road, Cottingham, HU16 5JQ, UK. E-mail: ahmedfahim@doctors. org.uk

Statement of Interest: None declared.

\section{REFERENCES}

1 Everett CF, Morice AH. Clinical history in gastroesophageal cough. Respir Med 2007; 101: 345-348.

2 Lee JH, Barich F, Karnell LH, et al. National Cancer Data Base report on malignant paragangliomas of the head and neck. Cancer 2002; 94: 730-737.

3 Manolidis S, Shohet JA, Jackson CG, et al. Malignant glomus tumours. Laryngoscope 1999; 109: 30-34.

4 van den Berg R, Wasser MN, van Gils AP, et al. Vascularization of head and neck paragangliomas: comparison of three MR angiographic techniques with digital subtraction angiography. AJNR Am J Neuroradiol 2000; 21: 162-170.

5 Kliewer KE, Wen DR, Cancilla PA, et al. Paragangliomas: assessment of prognosis by histologic, immunohistochemical and ultrastructural techniques. Human Pathol 1989; 20: 29-39.

6 Mayer R, Fruhwirth J, Beham A, et al. Radiotherapy as adjunct to surgery for malignant carotid body paragangliomas presenting with lymph node metastases. Strahlenther Onkol 2000; 176: 356-360.

7 Netterville JL, Jackson CG, Miller FR, et al. Vagal paraganglioma: a review of 46 patients treated during a 20 year period. Arch Otolaryngol Head Neck Surg 1998; 124: 1133-1140.

DOI: $10.1183 / 09031936.00064309$ 